practice of appealing to the laity without the intervention of the medical or of the pharmaceutical profession. They describe symptoms and they denominate maladies in which they claim efficacy for their product. The logical outcome is self-prescription, which entails to the physician and to the druggist a certain loss and to the lay user a more serious damage. Little need be said as to this class of manufacturers; their position is known and the results are recognized.

The second class is less open and is far more insidious in its operations. It makes the public profession that it does not appeal to the laity; that its efforts are expended on the practitioner in close restriction. Its remedies are uniformly in the guise of strict medical nomenclature. It forwards its literature to the doctor and asks him to use its goods in prescribing. In this it makes him the agent to his own undoing and to the crippling the druggist, his assistant. In practice the advantages of these products are always more or less publicly made known to the laity. The prospectuses continue to bear the label "For the Doctor Only," but the private person into whose hands they fall thinks all the more by reason of the restricting label that he is getting true wisdom, that he becomes "even as the gods, knowing good and evil." Even if that does not happen the result becomes the same. Suppose the instance where the physician includes in his prescription some one of the products of this sort of manufacture; the patient learns from the bottle what to take and for the future he takes it without reference to the physician. The progress to self-prescription is only a little delayed, it is none the less inevitable.

Now, these are conditions which must be met by the profession. It is not that an appeal is made to the mercenary side, although the member of the profession is entit]ed to his honorarium. It is that as a profession having in its keeping the public health, it must face the dangers arising from the unskilled use of medicines.

It is not the intention to comment on this condition which has arisen without indicating the remedy. The professional integrity of the physician, as well as his personal interest, summons him to meet this newly arisen condition, and to meet it now while it is as yet in the formative stage. The remedy proposed is specification.

'The druggist is as yet uncertain of his position. His desire leads him to remain as the valued assistant of the medical profession, to regard his prescription department as the conter of his activity. For the moment he is hesitating between his profession and the allurements of commercialism. In this contingency the honest retail druggist welcomes specification by the physician; it serves to anchor him to his noble profession. If a prescription brought to him to be filled calls for essence of pepsin he may look at it in two ways. As a commercial man he will supply the essence of pepsin which is the cheapest to him to buy and which will when dispensed yield him the correspondingly larger profit. But if the prescribing physician will only show that he has made examination into the quality of the various pepsins, and has taken the pains to specify one make, the druggist is gladly freed from the responsibility of the choice and dispenses with professional satisfaction that which he finds specified in the prescription which he is filling. In the same way when the prescription calls for cascara sagrada. As a commercial man he knows that there are a score of cascaras of varying strength, but if the physician will but specify the product of a certain house, the druggist knows that the effect expected by the physician will in all human probability follow.

The druggist will gladly welcome specification. The physician should regard it as a duty. That brings the tinal consideration back to the saddlebags in which it began. 'The saddlebags of the old-time physician represented his own dispensing agency. Nothing went into them unless he had convinced himself of its efficacy. There are no longer saddlebags, but the duty is the same, the physician must certify in some way to the therapeutic value of the elements which go into the prescription which he writes. The responsibility is his, not the druggist's; he is the one to assure himself of the value of the ingredients, the druggist is but ancillary. The only way to accomplish this is by specification in prescriptions. It will avoid the Scylla of leading the laity to the wreck of self-prescription, and equally shun the Charybdis of inefficient prescriptions.

\section{CONTINENTAL VIEWS OF ALCOHOL IN THERAPEUTICS.*}

BY T. D. CROTHERS, M.D.

EDITOR JOURNAL OF INEERIETY, ETC.

HARTFORD, CONN.

The impression prevails that alcohol is used unquestioned as a drug in all the wine and beer making countries of Europe. A therapeutist and author recently wrote that "only in America and among a few cxtremists is there a doubt of the value of alcohol as a remedy."

I propose to give a brief review of the revolution of practice and theory concerning alcohol in medicine now going on in Europe. Thirty years ago the general principle of practice was stimulation. Alcohol was supposed to rouse up and support vital forces in disease. Twenty-three years ago the first practical denial was put into a permanent position in a public hospital in London, where alcohol was seldom or never used. At first its use was confined to certain extreme cases, then pure alcohol of a definite strength, reduced in water, was administered. Finally, it was abandoned and is now rarely used, and to-day, after twenty-five years, the hospital statistics show a lower rate of mortality. In 15,224 cases under care during this time, the mortality has been less than 7 per cent., much less than in other I condon hospitals where alcohol has been frecly used. This has been considered a practical demonstration, and has influenced medical theories largely in England.

Dr. Richardson's researches showing the anesthetic nature of alcohol have had a great influence in changing medical practice in England. The result has been seen in the rapid decrease of the spirit bills of public hospitals, and the abandoning of the spirit rations in the army and navy.

On the Continent, a number of scientific workers have published researches confirming Dr. Richardson's conclusions and bringing out other facts as to the action of alcohol on the brain and nervous system. These papers and the discussions which followed have been slowly working their way into the laboratory and hospital, and have been tested and found correct, materially changing current opinions and creating great doubts of the value of alcohol.

In $18 \% 6$ the prosecution of Dr. Hirschfeld, a Magde-

* Presented to the Section on Materia Medica, Pharmacy and Therapeutics, at the Fiftieth Annual Meeting of the American Medical Association, held at Columbus, Ohlo, June 6-9, 1899. 
burg physician, in the German courts, for not using alcohol in a case of septicemia, seemed to be the central point for a new demonstration of the danger of the use of alcohol in medicine. Dr. Hirschfeld was acquitted on the testimony of a large number of leading physicians from the large hospitals and universities of Europe. It was proved that alcohol was not a remedy which was specifically required in any disease; also that its value was most seriously questioned as a general remedy by many able men, and its substitution was practical and literal in most cases. Statistics were presented proving that alcohol was dangerous and never a safe remedy, and laboratory investigations confirming and explaining its action were given. Since then a sharp reaction has been going on in Europe, and alcohol is rapidly declining and passing away as a common remedy.

The physiologists have taken up the question of the action of alcohol on the body and brain and, curiously enough, a great many have announced themselves as total abstainers and have become very prominent in the condemnation of the use of alcohol, both as a beverage and common drug. Some of these may be mentioned, as Professors Soule and Folel of Zurich, Switzerland; Hersten of Lausanne; Bung of Basle and Schiff of Geneva; McKenzie, Horsley, Gould, Woodhead and others of England. The list of eminent teachers and specialists of Europe who have written against the use of alcohol as a drug, except in some unusual cases, is growing rapidly, and the lists of papers in the Index Medicus and the German publications of this class are startling evidence of this change.

The increased action of the heart, with the feeling of warmth and exhilaration which follows the use of alcohol, has always been considered evidence of its medicinal value. The diminution and cessation of pain, with the relief and buoyancy which followed have been interpreted by both laymen and physicians as stimulation. Yet, certain phenomena appeared that were unexplainable. 'Thus, the depressions of vital force, acute intlammatory states, sudden death from heart failures, profound anemias and other symptoms have been attributed to the quality and dosage of the alcohol. 'These gave rise to minute studies and directions concerning its use, and cautions as to when and where it should be given. Elaborate experiments were made on the physiologic effect of alcohol on the organism of animals, in the laboratory, the results of which have varied and are unsettled even up to the present time.

It was not until the subject was taken up psychically, and: the phenomena were tested and examined by instruments of precision, that the real facts became apparent. Richardson began years ago, and many others have continued the research, and at present a large mass of facts has accumulated, some of which may be stated as follows:

The most common of all symptoms is the increased heart action, which is found to be four thousand beats in twenty-four hours for every ounce of alcohol used. This is found to be, not the outcome of a new force, but the calling into activity of the reserve powers and force of the heart. The heart action normally is the result of arterial pressure and nervous action. The latter is diminished by the narcotic action of alcohol and the former deranged. The flushed face following the increased activity of the heart is due to loss of nerve control, calling into action the reserve heart force. This is clear from the diminished force, measurable by instruments. The more rapid the heart beats, the weaker it becomes, so that alcohol is first an irritant, then a depressant and paralyzer. 'This is also proved by the symptoms of exhaustion which follow, and muscular measurment. Destree of Brussels concluded, as the result of most elaborate studies, that alcohol on the heart and muscular power has at first a slightly favorable effect, but a very transient one. 'The heart's force begins to decline at once, depending on the amount of spirits used. The muscular power reaches its maximum in thirty or forty minutes, and after that it is with difficulty kept up. The paralyzing effect increases, and its measurement becomes more and more exact and certain. Literally, alcohol does not supply any new energy to the body, but liberates the existing stock of energy with greater loss and exhaustion.

The action of alcohol on the stomach is physiologic, and not well understood. If in large doses, muscular contractibility is arrested, flaccidity and dilation result. Irigestion is interfered with, the food is passed on partially digested and undergoes putrefactive decomposition. Sensation is blunted, and satiety is lost. The action of alcohol on the senses and mental phenomenon has been examined with much exactness. Here instruments of precision vield similar results, and but little difference of opinion prevails. Paralysis of all the special senses follows; the functional activity of the brain is lessened down to complete narcotism. Forel of Zurich has been able to note these changes following the use of 20 c.c. of alcohol. This is the smallest dose the effects of which have been measured. Larger doses and doses taken at regular intervals show this same paralyzing action.

The conclusion that all authorities agree on is that alcohol is always a depressant and anesthetic. So far these anesthetic effects are found to appear very soon after spirits are used, and to follow a certain uniformity of progress depending on the conditions and dose. These facts are being rapidly increased and confirmed by both clinical and laboratory observations. Chloroform, sulphuric ether, chloral and other well-known anesthetics, all exhibit, in an extreme degree, the physiologic action of alcohol.

The value of alcohol in disense has been and is seriously questioned in the minds of many persons. Tradition, social custom, and empiric dogmatism have invested the question with difficulties which, happily, are fast disappearing. A number of authorities have enumerated the diseases and conditions of disorders in which alcohol is counterindicated. This list has now grown to such an extent as to practically include, almost every condition of disease and degeneration known. Dr. Clouston is very emphatic in showing the danger of alcohol to all who have suffered from head injuries and inflammation of the brain and other nerve affections.

Professor Woodhead, the Cambridge pathologist, gives the following list of conditions in which it should not be used. In those: 1 . Who have any family history of drunkenness, insanity, or nervous disease. 2. Who have used alcohol to excess in childhood or youth. 3 . Who are nervous, irritable, or badly nourished. 4. Who suffer from injuries to the head, gross disease of the brain, and sunstroke. 5. Who suffer from great bodily weakness, particularly during convalescence from exhausting diseases. 6. Who are engaged in exciting or exhausting employment, in bad air and surroundings in workshops and mines. $\%$. Who are solitary or lonely, and require amusement. 8 . Who have little self-control, either hereditary or acquired. 9. Who suffer from 
brain weaknesses, the result of senile degeneration. 10 . Who suffer from organic or functional diseases of the stomach, liver, kidneys or heart. 11. Never to be given to young children and in the adolescent stage.

This list is practically prohibitive of all use of alcohol in medicine. At all events, it indicates that its use rnust be based on new facts, and on exact application of means to accomplish a certain end. While laboratory researches continue to bring out the anesthetic effects and paralyzing action of alcohol, clinical observations, first of all, show that there are many substitutes and other remedies whose action is equally valuable with that of alcohol. The supposed stimulation is irritation and narcotism. No new force is added, but large demands on the reserve force are made. A new bacterial toxin is added to the blood, oxidation, elimination and digestion are diminished and, finally, seriously and measurably impaired. These facts are accepted as fully established, and the disputed points are in what doses are these effects seen to follow, and are they due to the quantity and quality of the alcohol given? Are they only certain physiologic poison effects, and are different results bound to follow smaller doses given in a certain way? Dr. Frick, an eminent teacher of medicine in Zurich, Switzerland, and Dr. Van Speyer of the University of Berne, have become very prominent in their writings against the use of alcohol in medicine except in rare and peculiar conditions. They have made statistical studies of cases treated with and without alcohol, and have analyzed the effects of spirits as medicinal agents to check and antagonize disease, and assert, very positively, that alcohol is a dangerous and exceedingly doubtful rernedy. Dr. Meyer of the University of Gottenburg, Dr. Moeleius of the Medical School of Theipsic, and Dr. Wehberg of Dusseldorf are equally prominent physicians who have taken the same position and are equally emphatic in their denunciations of the current beliefs concerning alcohol in medicine.

These and many other physicians have organized two societies for the study of the alcohol question on the same plan as the English Medical Temperance Society and the American Medical Temperance Society, the only difference being that the two latter societies are cornposed exclusively of physicians, while the Swiss and German societies comprise laymen, teachers and physicians. A French society, with a large membership of medical men, has been in existence for ten years. These societies have been making total abstinence the central object of their work, to which is added new studies of alcohol, and new theories of its value as a medicine. Both the English and American society have confined their work to the physiologic and therapeutic action of alcohol, while the German, Swiss and French societies have taken up the hygienic, sociologic and statistical sides of the subject.

In Russia, Sweden, Denmark, Austria and Italy, similar societies have been formed, with physicians as leaders and the scientific study of alcohol as the central theme. The result has been that the medical profession has become far more interested than laymen. This is the opposite of public sentiment in England and America. Here the alcohol question and its study is confined very largely to laymen, and to the hygienic and sociologic sides. On the Continent, where physicians are prominent, the physiology, pathology and therapeutics are the leading topics. Revolutions and changes in scientific practice go slow in Europe. A drug like alcohol, which has been in use as a beverage so long, and also as a domestic remedr and supposed tonic and stimulant, can not be changed and driven out in one generation.
Its domestic use in lisurope is more strongly intrenched in the customs and prejudices of the people than in this country, but its therapeutic use has never been so firmly fixed in practice as in England and America. For that reason physicians on the Continent have less personal bias to the therapeutic use of alcohol. They are more disposed to question its claims and test its value.

The brilliant discoveries in pathology and psychology have brought out the fact that alcohol, next to syphilis, is one of the most dangerous poisons in its effects on the body. This is rousing new and critical inquiries about the theories of its value in medicine. These reveal the errors of the value of alcohol in medicine. The test of clinical experience confirms the conclusions of pathology and physiology. As a result, Continental physicians are rapidly changing their views of alcohol in therapeutics and questioning the theories on which its use is based. Doubts concerning the place of alcohol in medicine are rapidly increasing in all German schools of medicine, and the physicians are recognizing this change in their practice.

In this country several elaborate experiments have been made treating cases without alcohol, and the results have fully confirmed the theories of Continental physicians. While alcohol is used, and in many cases very freely, there is a critical spirit abroad and the current literature is full of doubts and denials. The defenders of alcohol in therapeutics are disappearing, and reference to spirits as a tonic or stimulant are timidly made and feebly supported in the journals.

The question is very tersely put by Dr. Baere of Berlin, who says, in substance: The time has come for a change of theory and practice concerning alcohol in medicine when modern pathology, chemical and psychologic research, all fail to support the theories on which alcohol is used in medicine. Dr. Lagand of Paris puts the same idea in another form when he says: Our previous conceptions of alcohol and its action on the body are contradicted by clinical experience and chemical experiment in the laboratory." From a pathologic point of view, alcohol is shown to be one of the most insidious and destructive of tissue poisons, and its use is followed by certain cell and tissue degenerations that are uniform in their progress and growth. The theory of a tonic and stimulant value, or a producer or force conserver can not be sustained by any facts that are unquestioned.

The conclusions are inevitable, that alcohol and its theories as a therapeutic drug must be modified, and its use in medicine will change and, no doubt, may be put aside as worthless and dangerous in the near future.

\section{MODERN THERAPEUTICS.* BY WARREN B. HILL, M.D. \\ MILWAUKEE, wis.}

There is no field in medicine that presents such opportunities for exploration as therapeutics. There is none in which so much work has been done and none where such results may be expected. Therapeutics is being revolutionized. The biologic laboratory has become one of the important factors in the preduction of our remedies. It not only furnishes us with the various serums and animal extracts now so universally used, but it also affords us a means of standardizing vegetable products so that the very best results may be attained.

The most marked advance in therapeutics is shown

* Presented to the Section on Materia Medica, Iharmacy and Therapeutics, at the Fiftieth Annual Meeting of the American Medical Association, held at Columbus, Ohio, June 6-9, 1899. 